\title{
Effects of Dimethyl Sulfoxide on the Pluripotency and Differentiation Capacity of Mouse Embryonic Stem Cells
}

\author{
Jun-Koo Yi, ${ }^{1, *}$ Song Park, ${ }^{2}$ Jae-Jung Ha, ${ }^{1}$ Dae-Hyun Kim, ${ }^{1}$ Hai Huang, ${ }^{3}$ Si-Jun Park, ${ }^{4}$ \\ Mee-Hyun Lee, ${ }^{5,6}$ Zae-Young Ryoo, ${ }^{4}$ Sung-Hyun Kim, ${ }^{7}$ and Myoung-OK Kim ${ }^{3, * *}$
}

\begin{abstract}
Mouse embryonic stem cells (mESCs) go through self-renewal in the existence of the cytokine leukemia inhibitory factor (LIF). LIF is added to the mouse stem cells culture medium, and its removal results in fast differentiation. Dimethyl sulfoxide (DMSO) is one of the most used solvents in drug test. We exposed 4-day mESC cultures to different concentrations of $\operatorname{DMSO}(0.1 \%, 0.5 \%, 1.0 \%$, and $2.0 \%)$ to identify the safest dose exhibiting efficacy as a solvent. mESCs grown under general pluripotency conditions in the absence of LIF were treated with DMSO. In addition, as a control for differentiation, mESCs were grown in the absence of LIF. DMSO upregulated the mRNA expression level of pluripotency markers. Moreover, DMSO reduced the mRNA expression levels of ectodermal marker ( $\beta$-tubulin3), mesodermal marker (Hand1), and endodermal markers (Foxa2 and Sox17) in mESCs. These results indicate that DMSO treatment enhances the pluripotency and disrupts the differentiation of mESCs. We also show that members of the Tet oncogene family are critical to inhibiting the differentiation and methylation of mESCs. DMSO is appropriate to sustain the pluripotency of mESCs in the absence of LIF, and that mESCs can be sustained in an undifferentiated state using DMSO. Therefore, DMSO may, in part, function as a substitute for LIF.
\end{abstract}

Keywords: mESCs, DMSO, LIF, pluripotency markers

Introduction

D IMETHYL SULFOXIDE (DMSO) is a usual industrial solvent with amphiphilic properties, which can solvate a widespread substances. Although, several studies have shown that DMSO can promote differentiation of various cells, including embryonic teratocarcinoma cells and embryonic stem cells (ESCs) (Choi et al., 2015; Jacob and Herschler, 1986). Also, several studies have demonstrated the use of DMSO treatment on human and mouse embryonic stem cells (mESC) for maintaining pluripotency status and promoting differentiation upon removal of DMSO treatment by regulating the cell cycle of pluripotent stem cells (Chetty et al., 2013; Li et al., 2018; Shcherbina et al., 2019).
mESCs have pluripotency and unlimited capacity for differentiation and self-renewal. Pluripotency markers (Oct4, Sox2, Lin28, Nanog), and alkaline phosphatase (AP) are significant for sustaining the cells' pluripotency and selfrenewal capacity (Chambers and Smith, 2004; Chen et al., 2013; Wang et al., 2006). mESCs obtained from preimplantation embryos can be cultured in an undifferentiated state and are considered a suitable model for preimplantation toxicity test. ESCs have been generally used in the study of early embryonic state of pluripotent cell and development (Balmer et al., 2012; Moorthy et al., 2005; Sroczynska et al., 2009; Vallier and Pedersen, 2005).

On removal of leukemia inhibitory factor (LIF) and feeder cells grown on nonadhesive dishes, mESCs differentiate

\footnotetext{
${ }^{1}$ Department of Embryo Transfer Research, Gyeongbuk Livestock Research Institute, Yeongju, Korea.

${ }^{2}$ Core Protein Resources Center, DGIST, Daegu, Republic of Korea.

${ }^{3}$ Department of Animal Science and Biotechnology, Kyungpook National University, Sangju, Korea.

${ }^{4}$ Department of Life Sciences and Biotechnology, School of Life Sciences and Biotechnology, Kyungpook National University, Daegu, Korea.

${ }^{5}$ College of Korean Medicine, Dongshin University, Naju, Jeollanamdo, Korea.

${ }^{6}$ China-US (Henan) Hormel Cancer Institute, No. 127 Dongming Road, Zhengzhou, Henan, China.

${ }^{7}$ Life Medicine Analysis Korea Polytechnics Institute, Nonsan, Korea.

*First author.

***Correspondence author.
} 
into cells of three primary germ layers: the endoderm, mesoderm, and ectoderm. LIF is indispensable for maintaining mESC pluripotency. Removal of LIF allows stem cells to differentiate, but these cells retain their proliferative potential and pluripotency (Youngblood et al., 2014). Therefore, LIF is used in the culture of mESCs. LIF is necessary to sustain stem cells in an undifferentiated state. Although genetic manipulation of ESCs enables LIF-independent growth, particularly by overexpression of the gene Nanog (Ogawa et al., 2004), LIF is added to stem cell culture medium to decrease inherent differentiation (Kawahara et al., 2009).

DNA methylation is a stable epigenetic mark that is critical for diverse biological processes, including gene and transposon silencing, imprinting, and the suppression of retrotransposons (Bird, 2002). DNA demethylation comes about in the early embryo and the germ cells (Seisenberger et al., 2012; Smith et al., 2012) and may be mediated by the ten-eleven translocation (Tet) family of enzymes (Tet1,2,3) (Gu et al., 2011; Hackett et al., 2013; Yamaguchi et al., 2012), which convert 5-methylcytosine (mC) to 5hydroxymethylcytosine (hmC) (Tahiliani et al., 2009). Tet enzymes have been extensively studied in mESCs (Ficz et al., 2011; Pastor et al., 2011; Williams et al., 2011; Wu et al., 2011). In this study, we report that the addition of DMSO to mESCs promotes Tet activity, leading to an increase in the number of mESCs.

In the present study, we developed a novel and robust method for the differentiation of mESCs into the three germ layers and evaluation of their methylation potential by the addition of DMSO. DMSO has diverse applications in cell culture (Friend et al., 1971; Hollebeeck et al.; Pal et al., 2012; Singh et al., 2012; Thaler et al., 2012; Yoshizawa et al., 1997), as it is used to maintain the function of cultured hepatocytes in vitro (Su and Waxman, 2004) and to generate mESC-derived hepatic progenitor/hepatoblasts (Hay et al., 2008). We added DMSO to an LIF-based medium during differentiation to efficiently and concertedly downregulate mESC pluripotency genes and upregulate germ layer markers, and to study the effects of DMSO on DNA methylation potential in mESCs.

\section{Materials and Methods}

\section{mESC culture and differentiation}

E14 mESC line was cultured in serum-free Advanced ${ }^{\circledR}$ DMEM (Invitrogen UK Ltd., Paisley, UK) containing 10\% FBS, $1 \%$ penicillin/streptomycin, and $1000 \mathrm{U} / \mathrm{mL}$ LIF or absence of LIF (Millipore, Nottingham, UK) as previously described. Cells were passaged every 3 days and plated at a concluding mass of $5000 \mathrm{cells} / \mathrm{cm}^{2}$ for all culture experiments (Rodrigues et al., 2015). To promote differentiation, ESCs were cultured in the presence (pluripotency control) or absence (differentiation control) of LIF and with various doses of DMSO plus LIF. Thus, cells in the DMSO conditions were likened to mESCs cultured with or without LIF. Further details of the DMSO experiments are shown below.

\section{DMSO treatment}

E14 mESCs were used to evaluate the dose-dependent effect of DMSO ( $\geq 99.7 \%$; Sigma-Aldrich Co. LLC) to assess the solvent toxicity at room temperature (RT). DMSO was diluted in culture medium to final concentrations of $0.1 \%, 0.5 \%, 1.0 \%$, and $2.0 \%(\mathrm{v} / \mathrm{v})$, corresponding to low, medium, and high doses, respectively. DMSO was applied for 96 hours to cells in suspension, and after plating, the effects of the various doses of DMSO compared with the controls (with and without LIF) were evaluated.

\section{Cell cycle analysis}

E14 mESCs were seeded in a six-well cell culture plates and cultured for 96 hours. The cells were harvested by trypsinization, fixed with ethanol, stained with propidium iodide, and then analyzed for cell cycle phase by flow cytometry.

\section{Cell viability assay}

E14 mESCs were seeded $\left(1 \times 10^{4}\right.$ cells/well $)$ in six-well cell culture plates and then treated with different concentrations of DMSO $(0.1 \%, 0.5 \%, 1.0 \%$, and $2.0 \%)$. The medium was changed every day. After incubation for 96 hours, count the cell by Trypan Blue, and calculate.

\section{AP assay}

E14 mESCs, which are pluripotency and self-renewing, produce high levels of AP (O'Connor et al., 2011). An Alkaline Phosphatase Detection Kit (Millipore) was used to assay AP activity following the manufacturer's instructions. Shortly, mESCs were cultured in six-well plates for 96 hours, medium was eliminated, and were fixed with $4 \%$ paraformaldehyde for 1 minute (Rodrigues et al., 2015). mESCs were washed and the AP reagent was added. After 20 minutes of incubation at RT in the dark, the reagent was removed and Dulbecco's phosphate buffered saline (D-PBS) was added. All colonies in the plates were counted. All experiments were performed in triplicate.

\section{RNA isolation and quantitative real-time polymerase chain reaction assay}

Total RNA was isolated using TRIzol reagent (Invitrogen) according to the manufacturer's instructions. RNA concentration and quality were used a NanoDrop 2000 spectrophotometer (Thermo Scientific), and samples with an absorbance at 260/280 $\mathrm{nm}$ ratio of $<1.8$ were discarded (Varum et al., 2009). Real-time polymerase chain reaction (PCR) for the quantification of gene expression was carried out on an ABI7900 Fast Real-Time System (Applied Biosystems) according to the manufacturer's instructions. Synthesis of cDNAs for coding genes were carried out using $1 \mu \mathrm{g}$ of total RNA according to the manufacturer's instructions (Takara). mRNA expression levels were surveyed by real-time PCR using SYBR Green PCR Master Mix reagent kits (Takara). Internal control used glyceraldehyde 3-phosphate dehydrogenase (GAPDH). The sequences of primers are shown in Table 1.

\section{Western blot assay}

The cell lysates were prepared with lysis buffer (150 mmol/L NaCl, $1 \%$ NP-40, $50 \mathrm{mM}$ Tris-HCl, with $1 \mathrm{mM}$ phenylmethylsulfonyl fluoride (PMSF) and protease inhibitor and dephosphorylate inhibitor tablets mixture). After centrifugation at $12,000 \mathrm{rpm}$ for 30 minutes, extract the 
Table 1. Real-Time Polymerase Chain Reaction Assay Primers Used to Semiquantitatively Analyze Gene Expression in E14 Mouse Embryonic Stem Cells

\begin{tabular}{llll}
\hline Markers & \multicolumn{1}{c}{ Gene } & \multicolumn{1}{c}{ Sense } & Antisense \\
\hline Pluripotency & Sox2 & TGC AGT ACA ACT CCA TGA CC & TGC GAG TAG GAC ATG CTG TA \\
& Oct4 & TCA CCC TGG GCG TTC TCT T & GGC CGC AGC TTA CAC ATG TT \\
& Lin28 & AGG GCT TGG AAT CTA TCC GA & TTT GCA TTC CTT GGC ATG AT \\
Endoderm & FoXa2 & GCT GAC GCT GAG CGA GAT CT & GAG AGT GGC GGA TGG AGT TC \\
& Sox2 & CGA ACA GTA TCT GCC CTT TGT G & CCG TGG CTG TCT GAG AGG TT \\
Mesoderm & Hand & GTG AGT GCA TCC CCA ATG TG & GCC AGC ACG TCC ATC AAG TA \\
Ectoderm & $\beta$-Tubulin3 & GAG TCA CCA CCT CCC TTC GA & AGC CGG GCA TGA AGA AGT G \\
Methylation & Tet1 & GAG AGA TTT CTC GGG TCA GCA T & TTC CTC CTC TCC ACC ATT GG \\
& Tet2 & TGT TGT TGT CAG GGT GAG AAT C & TCT TGC TTC TGG CAA ACT TAC A \\
& Tet3 & CCG GAT TGA GAA GGT CAT CTA C & AAG ATA ACA ATC ACG GCG TTC T \\
\hline
\end{tabular}

FIG. 1. Effects of DMSO on mESC morphology and viability. (A, B) E14 mESCs were exposed to DMSO, and cell morphology and viability were observed. Cells were cultured with various concentrations of DMSO $(0.1 \%, 0.5 \%$, $1.0 \%$, or $2.0 \%$ ) and for controls (with LIF or without LIF) for 96 hours. Magnification, $40 \times$. DMSO, dimethyl sulfoxide; LIF, leukemia inhibitory factor; mESC, mouse embryonic stem cell.
A
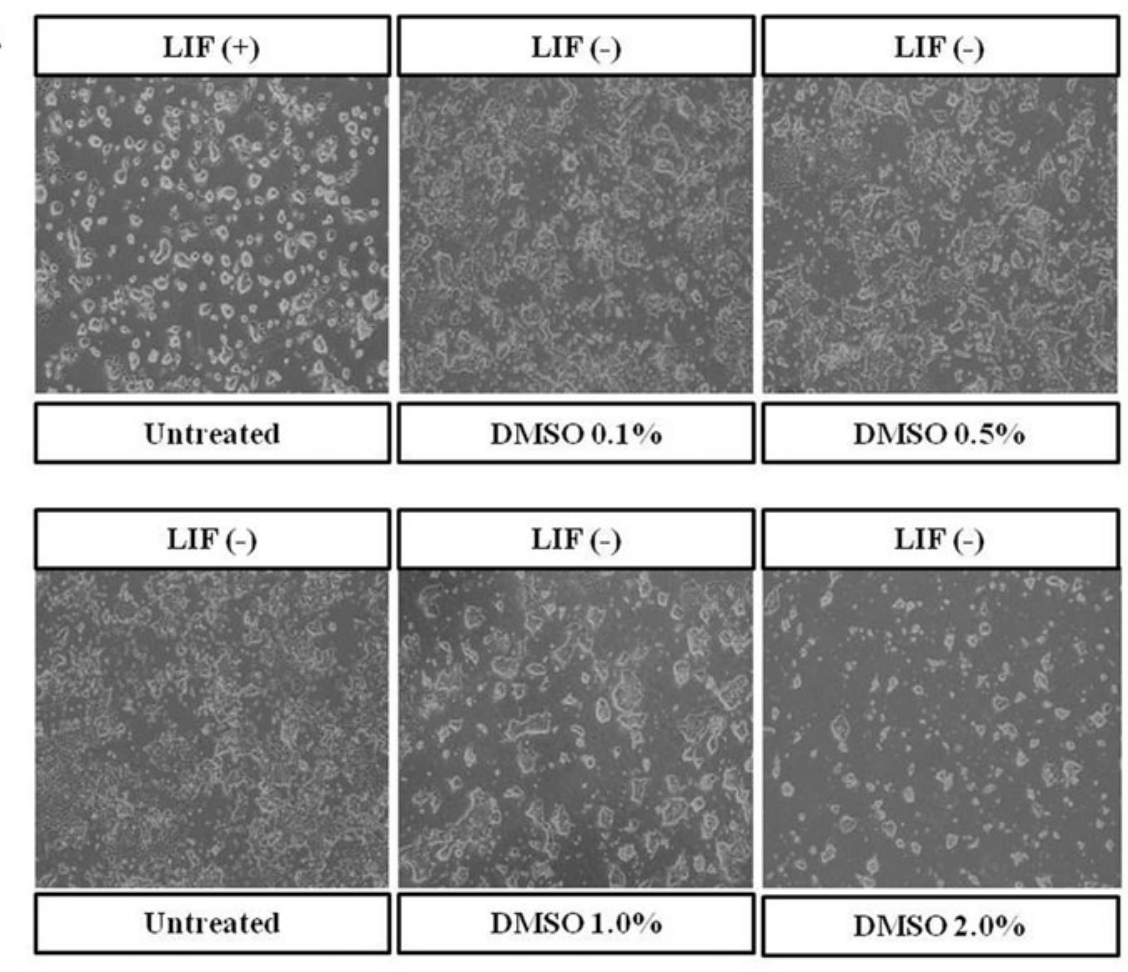

B
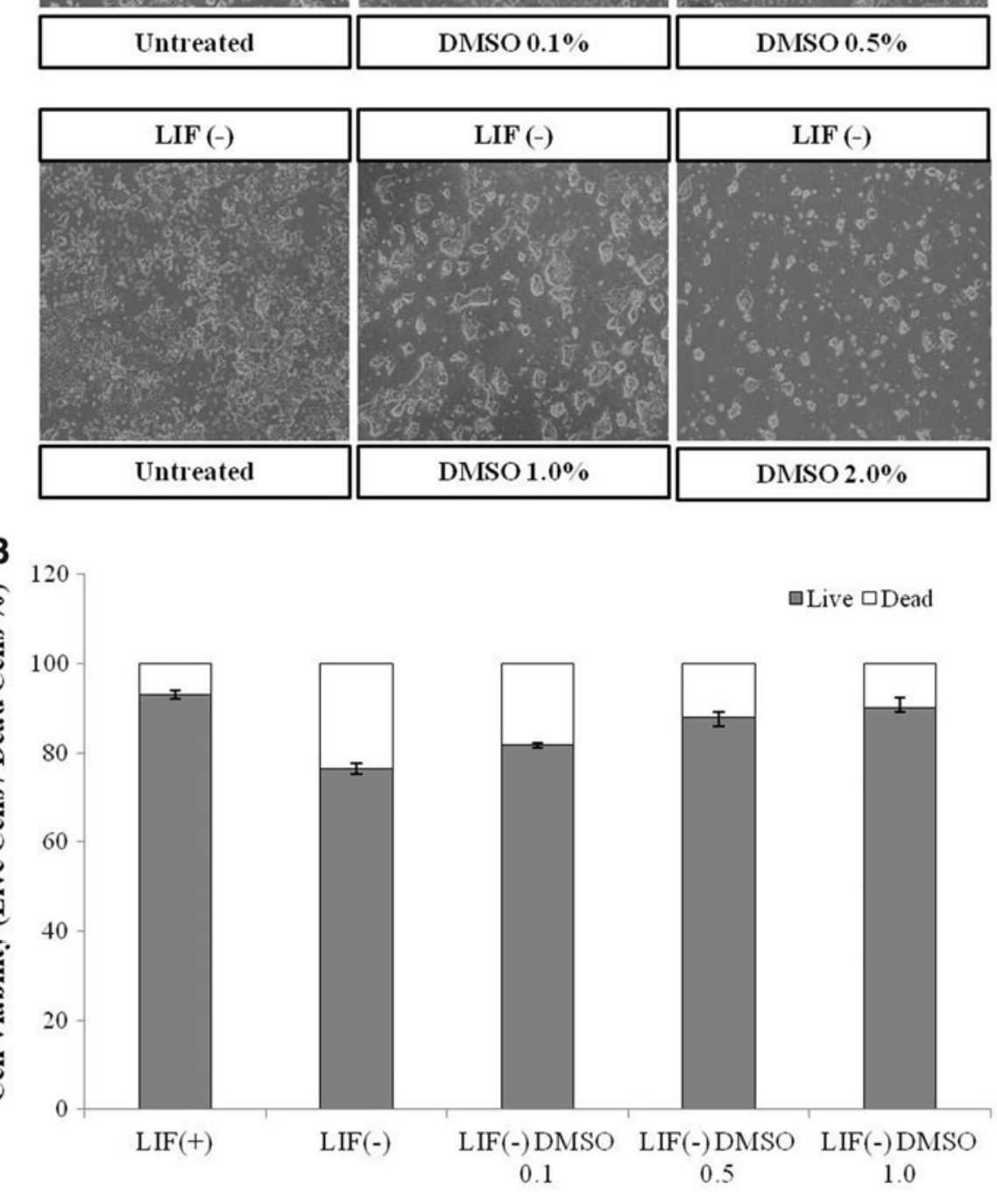
supernatant as total cellular protein. The concentration of protein was measured through the BCA Quantification Kit (Thermo). Proteins were separated by $8 \%-12 \%$ of sodium dodecyl sulfate polyacrylamide gel electrophoresis (SDSPAGE) and transferred to polyvinylidene difluoride membrane (Millipore, Billerica, MA). The membrane was blocked by $5 \%$ nonfat dry milk in $1 \times$ TBST for 1 hour and incubated with primary antibodies at $4^{\circ} \mathrm{C}$ for overnight, washing membrane five times with $1 \times$ TBST buffer and incubated with the correspondent secondary antibody for 1 hour.

The chemiluminescence substrate (ECL) detection reagent (GE Healthcare Life Science) was used to test protein bands through Da Vinci software. The antibodies against phosphorylated Oct-4 (\#2750) were purchased from Cell Signaling Technology (Beverly, MA), Anti-Nanog (ab80892) were purchased from Abcam (Beverly); and $\beta$-actin (sc-8432) were purchased from Santa Cruz Biotechnology (Santa, Cruz, CA).

\section{Statistical analysis}

Values are expressed as means \pm standard errors of the mean. Statistically significant differences between the DMSO treats $(0.1 \%, 0.5 \%, 1.0 \%$, or $2.0 \%)$ and controls were analyzed by analysis of variance, followed by Dunnett's multiple comparisons test. All tests of statistical significance were two-sided, and $p$-values $<0.05$ were considered to indicate statistical significance

\section{Results}

DMSO affects the morphology, viability, pluripotency, and cell cycle of mESCs

DMSO has been shown to regulate epigenetic modification by altering $\mathrm{CpG}$ methylation patterns in diverse E14 mESCs and tissues (Iwatani et al., 2006; Marks and Bre- slow, 2007), thus affecting their development and differentiation (Cheung et al., 2006). To study the effects of DMSO on cell morphology and differentiation, E14 mESCs were exposed to diverse concentrations of DMSO for 96 hours. mESCs were collected after 4 days of culture in the presence of diverse concentrations of DMSO $(0.1 \%, 0.5 \%, 1.0 \%$, or $2.0 \%$ ) or with the fundamental pluripotency factor LIF as a control for self-renewal and pluripotency. Colony growing as a round and dome shape could be seen under control conditions, whereas cells growing without LIF no longer formed these typical pluripotent colonies.

On the contrary, higher levels of differentiation were apparent, resulting in fewer colonies without defined borders. Rather, cultures grown without LIF in the presence of $1.0 \%$ and $2.0 \%$ DMSO produced well-defined, round colonies and exhibited a reduced number of differentiated cells in comparison with the differentiation control (without LIF) (Fig. 1A).

To explore the cell viability on the above identified effects of DMSO in E14 mESCs, cells were exposed to various concentrations of DMSO $(0.1 \%, 0.5 \%$, or $1.0 \%)$ or LIF as a control for 96 hours, and then collected after 4 days of culture. Treatment with $1.0 \%$ DMSO showed the highest rate of cell viability. Increased suppression of cell viability; the treat of $1.0 \%$ DMSO was $\sim 90 \%$, which was higher than that in control (without LIF) cells (Fig. 1B).

To determine whether DMSO influences pluripotency, we stained mESCs with AP. Differentiation of mESCs is characterized by the loss of AP staining and the emergence from a flattened cellular morphology (Ivanova et al., 2006). In the present study, mESCs treated with DMSO for 96 hours were resembling the control (with LIF), showing deep AP staining and a similar colony morphology (Fig. 2). Given that pluripotent cells express high levels of AP, we also performed an AP assay to monitor the number of APpositive red colonies (O'Connor et al., 2008, 2011) (Fig. 2).
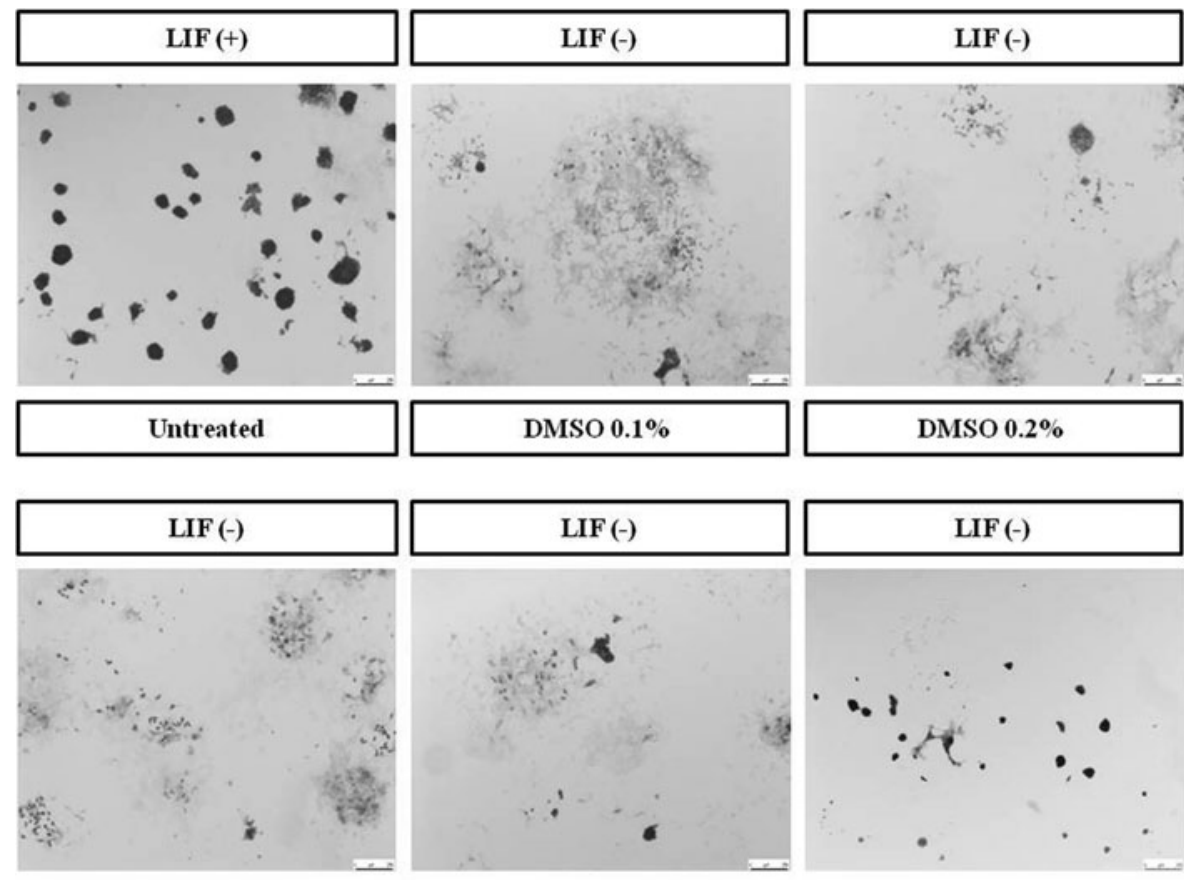

Untreated
DMSO $0.5 \%$

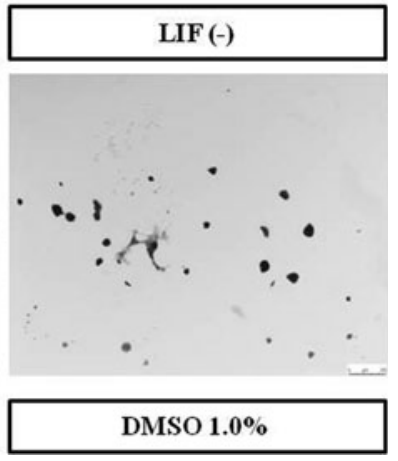

FIG. 2. Effects of DMSO on alkaline phosphatase staining and activity in mESCs. Cells were cultured with various concentrations of DMSO $(0.1 \%, 0.2 \%, 0.5 \%$, or $1.0 \%$ ) and for controls (with LIF or without LIF) for 96 hours. 


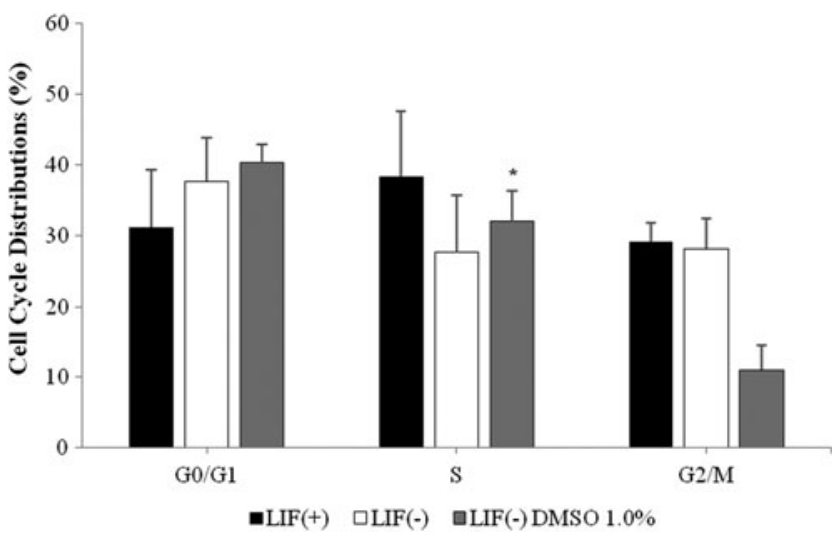

FIG. 3. Effects of DMSO on mESC cycle distribution. Percentage of cells in G0/G1, s and G2/M phase in $1.0 \%$ DMSO and for controls (with LIF or without LIF) for 96 hours. Each data point was normalized to the control (with LIF or without LIF) and represents the means \pm SE from three independent experiments. Indicates significant difference when the values were compared to that of the control $(p<0.05)$.
The differentiation control (without LIF) showed a low rate of AP-positive colonies, while cells growing without LIF in the presence of DMSO had a higher rate of AP-positive colonies. Treatment with $1.0 \%$ DMSO showed the highest rate of AP-positive colonies.

The effect of DMSO on cell cycle progression was analyzed by flow cytometry. The results suggest that DMSO induces $\mathrm{S}$ phase proportion in E14 mESCs compared to without LIF (Fig. 3). These findings indicate that DMSO is associated with cell cycle characteristics.

\section{DMSO regulates the expression of $\mathrm{mESC}$ pluripotency genes}

To determine whether DMSO influences mESC differentiation, cells were treated with DMSO and the effect on differentiation in the absence of LIF was evaluated. mESCs were exposed to various concentrations of DMSO $(0.1 \%$, $0.5 \%, 1.0 \%$, or $2.0 \%$ ) or LIF as a control for 96 hours, and then collected after 4 days of culture. The expression levels of pluripotency markers such as Oct4, Sox2, and Lin28 were
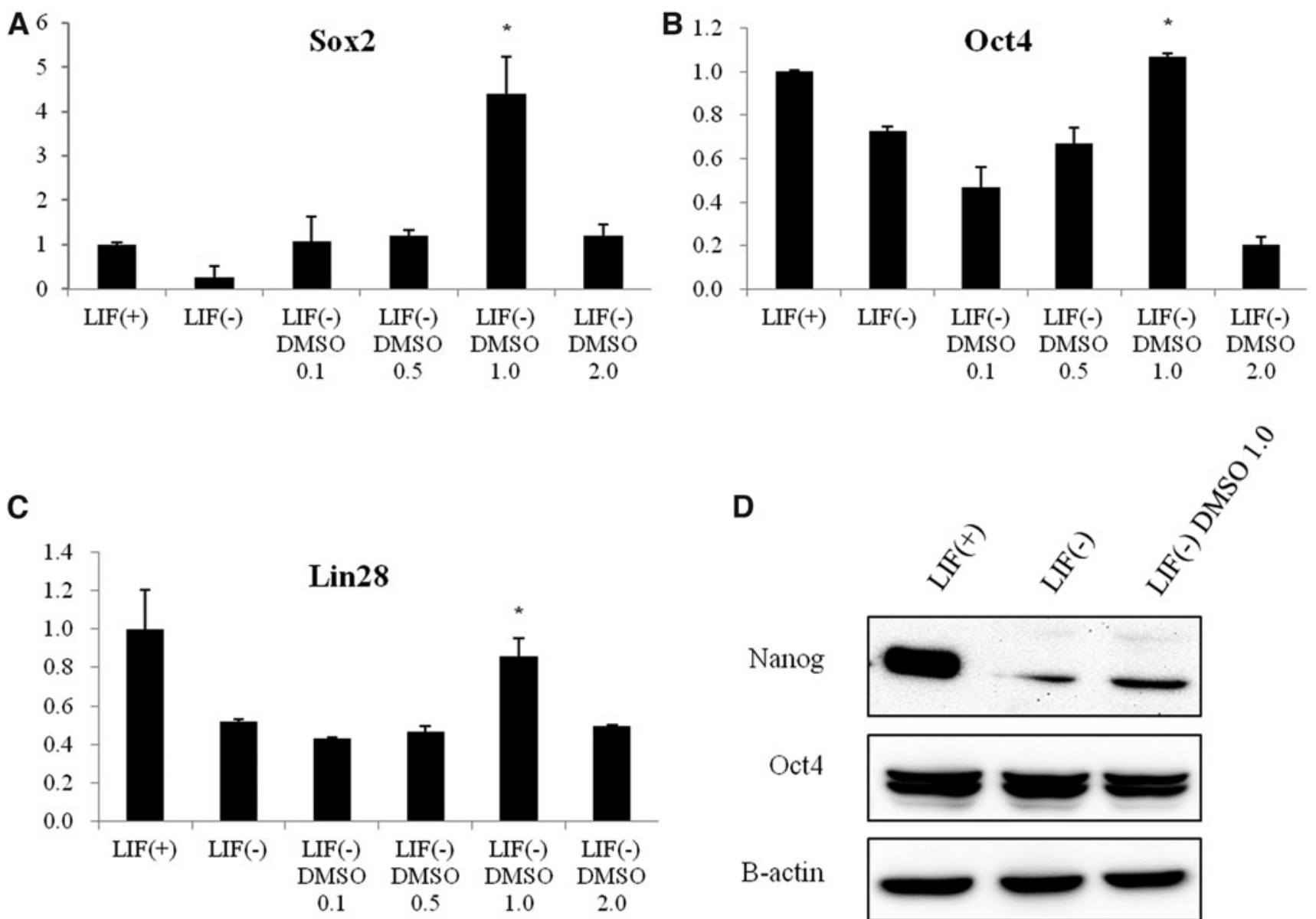

D

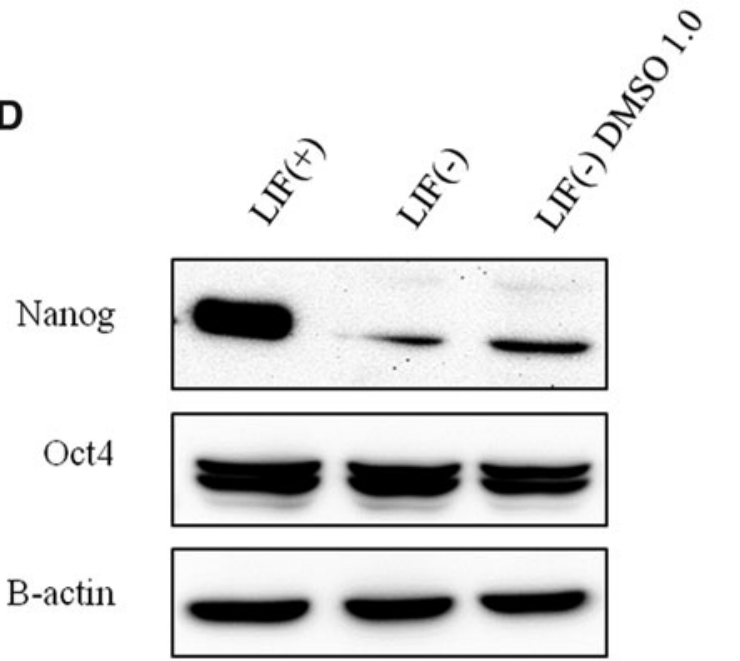

FIG. 4. Effects of DMSO on pluripotency and expression of Oct4, Sox2, and Lin28 in mESCs. Cells were cultured with various concentrations of DMSO $(0.1 \%, 0.5 \%, 1.0 \%$, or $2.0 \%)$ and for controls (with LIF or without LIF) for 96 hours. (AC) Oct4/Sox2/Lin28 mRNA levels were determined by quantitative real-time PCR using housekeeping gene GAPDH as an internal control. Each data point was normalized to the control (with LIF or without LIF) and represents the means \pm SE from three independent experiments. Indicates significant difference when the values were compared to that of the control $(p<0.05)$. Cells were treated $1.0 \%$ DMSO with LIF or without LIF. The expression of Nanog, Oct4, and $\beta$-actin proteins were determined by western blot assay (D). GAPDH, glyceraldehyde 3-phosphate dehydrogenase; PCR, polymerase chain reaction; SE, standard error. 
analyzed by quantitative real-time polymerase chain reaction assay (qRT-PCR) (Fig. 4A-C). Treatment with $0.1 \%$, $0.5 \%$, and $1.0 \%$ DMSO increased Oct4, Sox2, and Lin28 mRNA levels after 4 days in a dose-dependent manner. Treatment with $2.0 \%$ DMSO, however, resulted in a significant decrease in Oct4, Sox2, and Lin 28 mRNA expression levels after 4 days of culture.

We also confirmed that the expression levels of Nanog and Oct 4 were analyzed by western blotting, compared cells without LIF, and Nanog was also more increased by $1.0 \%$ DMSO, but expression of Oct4 was no difference (Fig. 4D).

mRNA expression levels of markers of endoderm, mesoderm, and ectoderm were also examined by qRT-PCR (Fig. 5A-D). mRNA levels of the endoderm marker Foxa2 decreased significantly when mESCs were treated with $0.5 \%$ or $2.0 \%$ DMSO (Fig. 4A). mRNA levels of another endoderm marker, Sox17, also decreased in a dose-dependent manner following DMSO treatment $(0.1 \%, 0.5 \%$, and $1.0 \%)$ (Fig. 5B). Treatment with $2.0 \%$ DMSO, however, significantly increased Sox 17 mRNA levels in comparison with those of the control. The mRNA levels for the mesoderm marker Hand 1 and the ectoderm marker $\beta$-tubulin 3 also decreased in a dose-dependent manner with DMSO treatment (Fig. 5C, D). On the whole, we find out that pluripotency
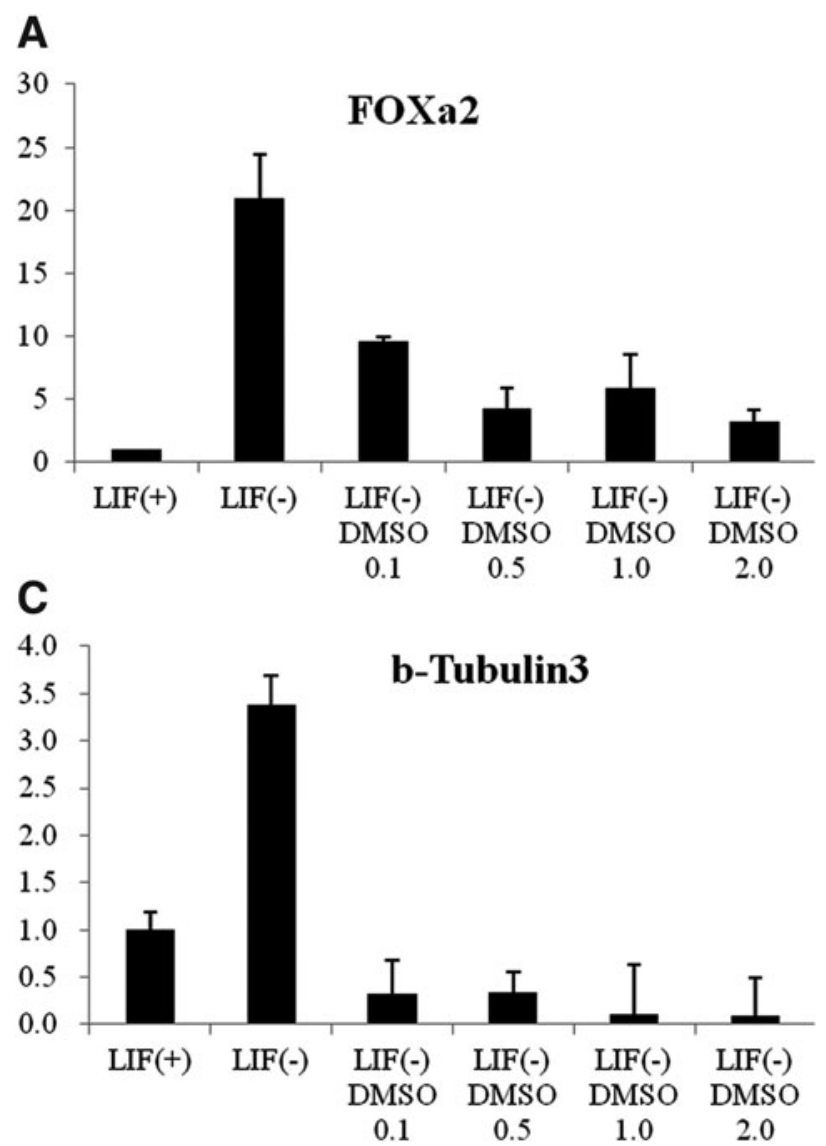

markers were differentially expressed, suggesting that DMSO biases differentiation toward the three germ layers.

\section{DMSO also affects the mRNA levels of genes involved in active DNA methylation}

To evaluate the effect of DMSO on the active DNA methylation of mESCs, qRT-PCR was used to assess mRNA expression levels of two members of the Tet oncogene family: Tet 1 (Tet oncogene 1) and Tet2 (Tet oncogene 2), following DMSO treatment. Expression of these genes after 4 days of culture increased in a dose-dependent manner; however, expression was downregulated by $2.0 \%$ DMSO treatment (Fig. 6A, B). mRNA expression of the third member of the Tet family, Tet3, was increased significantly in a dose-dependent manner by DMSO treatment (Fig. 6C). These findings suggest that regulating the expression of the Tet oncogene family members is critical for proper differentiation and methylation of mESCs.

\section{Discussion}

ESCs have properties like pluripotency, self-renewal, and continuous proliferation (Chambers and Smith, 2004; Nichols and Smith, 2011; Pera and Tam, 2010; Smith, 2001). For such properties, the mESCs can be maintained by the

FIG. 5. Effects of DMSO on differentiation capacity and expression of markers of three germ layers markers in mESCs (A-D). mRNA levels of endodermal markers (Foxa2, Sox17), mesodermal (Hand1), and ectodermal ( $\beta$-tubulin 3) markers were determined by quantitative real-time PCR using GAPDH as an internal control. Each data point was normalized to the control (with LIF or without LIF) and represents the means \pm SE from three independent experiments. Indicates significant difference when the values were compared to that of the control $(p<0.05)$. 

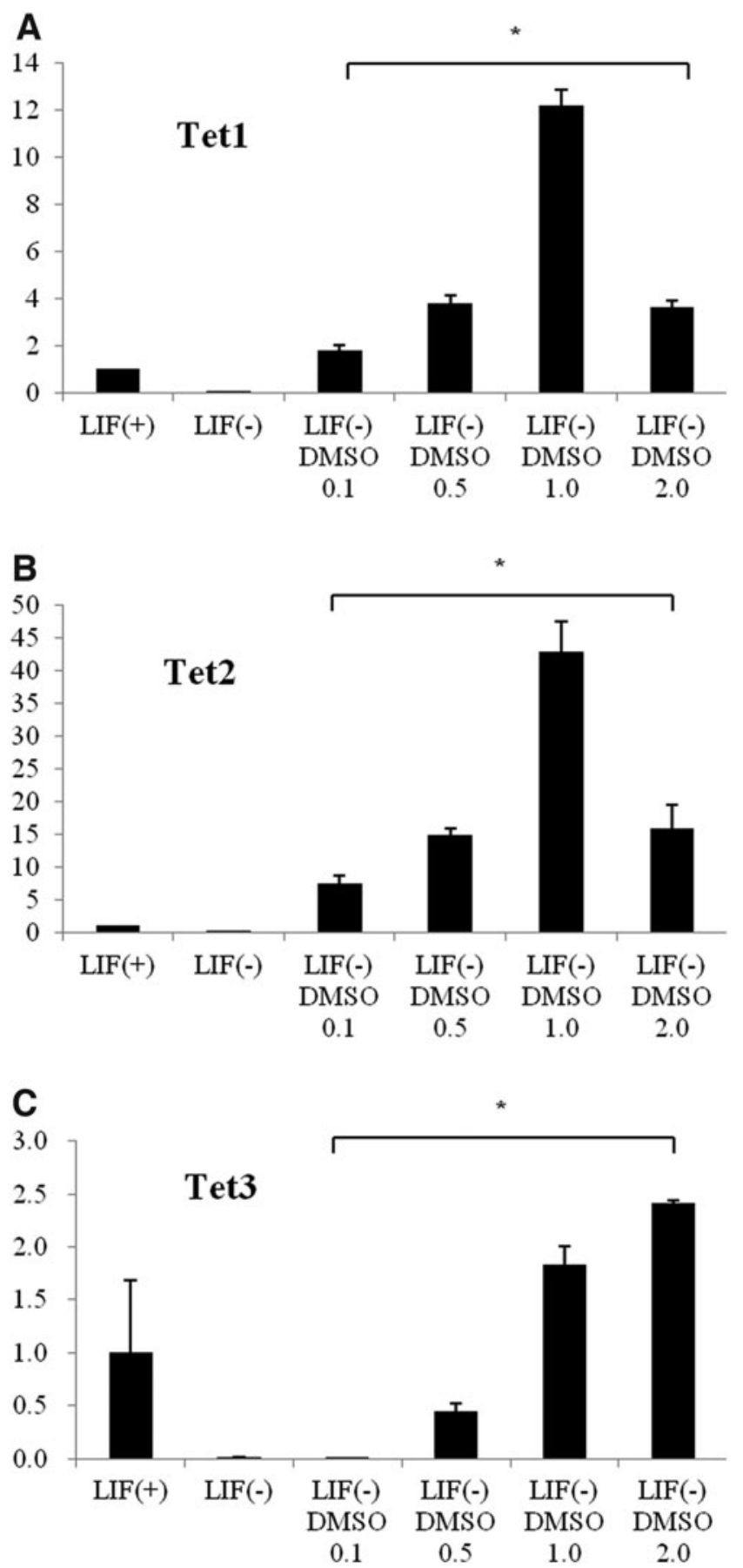

FIG. 6. Effects of DMSO on DNA methylation and expression of Tet oncogenes in mESCs (A-C). Tet1 (Tet oncogene 1), Tet2 (Tet oncogene2), and Tet3 (Tet oncogene 3 ) mRNA levels were determined by quantitative real-time PCR using GAPDH as an internal control. Each data point was normalized to the control (with LIF or without LIF) and represents the means $\pm \mathrm{SE}$ from three independent experiments. Indicates significant difference when the values were compared to that of the control $(p<0.05)$.

cytokine LIF, and LIF is regularly added to the culture of mESCs. Conversely, if LIF is not added, it brings the differentiation of mESCs. Because of such LIF properties, a lot of researchers have conducted the studies related to the ESCs, focused on such LIF properties with great interest.
DMSO is generally solvent for many different kinds of organic substance, and it is widely used for cryopreservation of cell as cryoprotectant. In particular, in case of culturing ESCs, DMSO regulates the differentiation of cell. This research came to conduct this study to examine the effect of solvent like DMSO on in vitro development (Jasmin et al., 2010). Especially, this study was really designed to investigate the effect of DMSO on the E14 mESCs and mouse embryonic body mEBs. The deduced result in this study showed the deeper understanding of the effect of DMSO on the early embryonic development. The treatment of DMSO increased the expression of pluripotency markers at mRNA level of E14 mESCs and decreased the expression of germ layer markers under $\mathrm{mEBs}$ condition.

Overall, according to our data, this study has found out that DMSO played an important role in regulating the pluripotency of mESCs and differentiation of mEBs through the regulation of expression for pluripotency markers (Oct4, Sox2, Lin28, and Nanog) related to the pluripotency (Tay et al., 2008a, 2008b).

It is important to understand the molecular mechanisms that control pluripotency and self-renewal in stem cells for application in the fields of developmental biology, regenerative medicine, and cancer biology. Pluripotency is sustained by a synergistic interaction between extrinsic stimulus and intrinsic circuitry, which allow for sustentation of an undifferentiated and self-renewing state.

Notwithstanding, despite recent study, the precise mechanisms regulating differentiation and self-renewal remain unclear. LIF is widely used for culturing the mESCs and IPSs and inhibiting the differentiation of cell by paracrine signal, while especially LIF stimulates the ESCs self renewal at mESCs (Gearing et al., 1987; Smith et al., 1988; Williams et al., 1988). The use of feeder cells is replaceable by adding LIF to culture solution, and serum is replaceable with BMP. Since such discovery, the condition of ESCs culture has greatly improved (Ying and Smith, 2003). However, it is still a difficult situation in the mechanism that DMSO affects mESCs, but this study has shown that DMSO could maintain pluripotency at mESCs without LIF.

Oct4, Sox2, Lin28, and Nanog are master modulators of pluripotency. Latest studies have suggested that Oct4, Sox2, Lin28, and Nanog form feed forward loops that maintain pluripotency of mESCs (Chen et al., 2008; Niwa, 2007). The expression of Oct4 governs the fate of ES cells, and regulation of this protein induces divergent developmental programs (Niwa et al., 2000). We report here that DMSO treatment upregulates Oct4, Sox2, and Lin28 in mESCs. In addition, DMSO specifically inhibits the expression of endodermal, mesodermal, and ectodermal lineage markers in mESCs. Our studies enhance understanding of DMSO and its effects of mouse embryonic development.

Defining the biological significance of the Tet family in development has been a primary focus of recent investigations. Oct4, Sox 2, and Nanog significantly raise the expression of Tet genes at an early stage of development, and the interaction of Tet with SOX2 and NANOG promotes efficient somatic cell reprogramming (Zhu et al., 2014). Interactions between Tet family members and pluripotency markers (Oct4, Nanog, and Sox2) have been observed in ESCs. In fact, Tet1 and Tet2 proteins were detected in pluripotency factor precipitation complexes (Zhu et al., 2014). Tet proteins could function with 
pluripotency markers in an ordinary pathway to control cell reprogramming and maintain mESC pluripotency.

We investigated whether DMSO treatment had any effect on active DNA methylation in mESCs and, specifically, on the interaction between Tet proteins and pluripotency markers (Oct4, Sox 2, and Lin28) in mESCs. As shown by the qRT-PCR analyses, DMSO affected mRNA expression of the Tet family of pluripotency genes involved in active DNA methylation.

\section{Conclusion}

We report the upregulation of pluripotency marker genes, including Oct4, Sox2, and Lin28, in mESCs upon DMSO treatment. In addition, DMSO treatment specifically regulated the expression of endodermal, mesodermal, and ectodermal markers and members of the Tet family in mEBs. These results improve our understanding of the effects of DMSO on mouse embryonic development and methylation. Overall, with further investigation, this study has the potential to be an interesting and important finding whereby DMSO treatment could be easily and widely applied for maintaining pluripotency and regulating differentiation of mESCs. Replacing LIF with DMSO would also be a costeffective strategy in mESC maintenance.

\section{Author Disclosure Statement}

The authors declare they have no conflicting financial interests.

\section{Funding Information}

This research was supported by the Basic Science Research Program of the National Research Foundation of Korea (NRF), funded by the Ministry of Education (2014R1A6A3A04059193) and Science and Technology (No. 2014R1A1A2059813). The funders had no role in study design, data collection and analysis, decision to publish, or preparation of the article.

\section{References}

Balmer, N.V., Weng, M.K., Zimmer, B., Ivanova, V.N., Chambers, S.M., Nikolaeva, E., Jagtap, S., Sachinidis, A., Hescheler, J., Waldmann, T., and Leist, M. (2012). Epigenetic changes and disturbed neural development in a human embryonic stem cell-based model relating to the fetal valproate syndrome. Hum. Mol. Genet. 21, 4104-4114.

Bird, A. (2002). DNA methylation patterns and epigenetic memory. Genes Dev. 16, 6-21.

Chambers, I., and Smith, A. (2004). Self-renewal of teratocarcinoma and embryonic stem cells. Oncogene 23, 7150-7160.

Chen, X., Xu, B., Han, X., Mao, Z., Talbot, P., Chen, M., Du, G., Chen, A., Liu, J., Wang, X., and Xia, Y. (2013). Effect of bisphenol A on pluripotency of mouse embryonic stem cells and differentiation capacity in mouse embryoid bodies. Toxicol In Vitro. 27, 2249-2255.

Chen, X., Xu, H., Yuan, P., Fang, F., Huss, M., Vega, V.B., Wong, E., Orlov, Y.L., Zhang, W., Jiang, J., Loh, Y.H., Yeo, C.H., Yeo, Z.X., Narang, V., Govindarajan, K.R., Leong, B., Shahab, A., Ruan, Y., Bourque, G., Sung, W.K., Clarke, N.D., Wei, C.L., and Ng, H.H. (2008). Integration of external signaling pathways with the core transcriptional network in embryonic stem cells. Cell 133, 1106-1117.
Chetty, S., Pagliuca, F.W., Honore, C., Kweudjeu, A., Rezania, A., and Melton, D.A. (2013). A simple tool to improve pluripotent stem cell differentiation. Nat. Methods. 10, 553-556.

Cheung, W.M., Ng, W.W., and Kung, A.W. (2006). Dimethyl sulfoxide as an inducer of differentiation in preosteoblast MC3T3-E1 cells. FEBS Lett. 580, 121-126.

Choi, S.C., Choi, J.H., Cui, L.H., Seo, H.R., Kim, J.H., Park, C.Y., Joo, H.J., Park, J.H., Hong, S.J., Yu, C.W., and Lim, D.S. (2015). Mixl1 and Flk1 are key players of Wnt/TGF-beta signaling during DMSO-induced mesodermal specification in P19 cells. J. Cell. Physiol. 230, 1807-1821.

Ficz, G., Branco, M.R., Seisenberger, S., Santos, F., Krueger, F., Hore, T.A., Marques, C.J., Andrews, S., and Reik, W. (2011). Dynamic regulation of 5-hydroxymethylcytosine in mouse ES cells and during differentiation. Nature 473, 398402.

Friend, C., Scher, W., Holland, J.G., and Sato, T. (1971). Hemoglobin synthesis in murine virus-induced leukemic cells in vitro: Stimulation of erythroid differentiation by dimethyl sulfoxide. Proc. Natl. Acad. Sci. U. S. A. 68, 378-382.

Gearing, D.P., Gough, N.M., King, J.A., Hilton, D.J., Nicola, N.A., Simpson, R.J., Nice, E.C., Kelso, A., and Metcalf, D. (1987). Molecular cloning and expression of cDNA encoding a murine myeloid leukaemia inhibitory factor (LIF). EMBO J. 6, 3995-4002.

Gu, T.P., Guo, F., Yang, H., Wu, H.P., Xu, G.F., Liu, W., Xie, Z.G., Shi, L., He, X., Jin, S.G., Iqbal, K., Shi, Y.G., Deng, Z., Szabó, P.E., Pfeifer, G.P., Li, J., and Xu, G.L. (2011). The role of Tet3 DNA dioxygenase in epigenetic reprogramming by oocytes. Nature 477, 606-610.

Hackett, J.A., Sengupta, R., Zylicz, J.J., Murakami, K., Lee, C., Down, T.A., and Surani, M.A. (2013). Germline DNA demethylation dynamics and imprint erasure through 5hydroxymethylcytosine. Science 339, 448-452.

Hay, D.C., Zhao, D., Fletcher, J., Hewitt, Z.A., McLean, D., Urruticoechea-Uriguen, A., Black, J.R., Elcombe, C., Ross, J.A., Wolf, R., and Cui, W. (2008). Efficient differentiation of hepatocytes from human embryonic stem cells exhibiting markers recapitulating liver development in vivo. Stem Cells 26, 894-902.

Hollebeeck, S., Raas, T., Piront, N., Schneider, Y.J., Toussaint, O., Larondelle, Y., and During, A. (2011). Dimethyl sulfoxide (DMSO) attenuates the inflammatory response in the in vitro intestinal Caco-2 cell model. Toxicol Lett. 206, 268-275.

Ivanova, N., Dobrin, R., Lu, R., Kotenko, I., Levorse, J., DeCoste, C., Schafer, X., Lun, Y., and Lemischka, I.R. (2006). Dissecting self-renewal in stem cells with RNA interference. Nature 442, 533-538.

Iwatani, M., Ikegami, K., Kremenska, Y., Hattori, N., Tanaka, S., Yagi, S., and Shiota, K. (2006). Dimethyl sulfoxide has an impact on epigenetic profile in mouse embryoid body. Stem Cells 24, 2549-2556.

Jacob, S.W., and Herschler, R. (1986). Pharmacology of DMSO. Cryobiology 23, 14-27.

Jasmin, Spray, D.C., Campos de Carvalho, A.C., and MendezOtero, R. (2010). Chemical induction of cardiac differentiation in p19 embryonal carcinoma stem cells. Stem Cells Dev. 19, 403-412.

Kawahara, Y., Manabe, T., Matsumoto, M., Kajiume, T., and Yuge, L. (2009). LIF-free embryonic stem cell culture in simulated microgravity. PLoS One 4, e6343.

Li, J., Narayanan, C., Bian, J., Sambo, D., Brickler, T., Zhang, W., and Chetty, S. (2018). A transient DMSO treatment 
increases the differentiation potential of human pluripotent stem cells through the Rb family. PLoS One 13, e0208110.

Marks, P.A., and Breslow, R. (2007). Dimethyl sulfoxide to vorinostat: Development of this histone deacetylase inhibitor as an anticancer drug. Nat. Biotechnol. 25, 84-90.

Moorthy, P.P., Kumar, A.A., and Devaraj, H. (2005). Expression of the Gas7 gene and Oct4 in embryonic stem cells of mice. Stem Cells Dev. 14, 664-670.

Nichols, J., and Smith, A. The origin and identity of embryonic stem cells. (2011). Development 138, 3-8.

Niwa, H. (2007). How is pluripotency determined and maintained? Development 134, 635-646.

Niwa, H., Miyazaki, J., and Smith, A.G. (2000). Quantitative expression of Oct-3/4 defines differentiation, dedifferentiation or self-renewal of ES cells. Nat. Genet. 24, 372-376.

O'Connor, M.D., Kardel, M.D., and Eaves, C.J. (2011). Functional assays for human embryonic stem cell pluripotency. Methods Mol. Biol. 690, 67-80.

O’Connor, M.D., Kardel, M.D., Iosfina, I., Youssef, D., Lu, M., Li, M.M., Vercauteren, S., Nagy, A., and Eaves, C.J. (2008). Alkaline phosphatase-positive colony formation is a sensitive, specific, and quantitative indicator of undifferentiated human embryonic stem cells. Stem Cells 26, 1109-1116.

Ogawa, K., Matsui, H., Ohtsuka, S., and Niwa, H. (2004). A novel mechanism for regulating clonal propagation of mouse ES cells. Genes Cells 9, 471-477.

Pal, R., Mamidi, M.K., Das, A.K., and Bhonde, R. (2012). Diverse effects of dimethyl sulfoxide (DMSO) on the differentiation potential of human embryonic stem cells. Arch Toxicol. 86, 651-661.

Pastor, W.A., Pape, U.J., Huang, Y., Henderson, H.R., Lister, R., Ko, M., McLoughlin, E.M., Brudno, Y., Mahapatra, S., Kapranov, P., Tahiliani, M., Daley, G.Q., Liu, X.S., Ecker, J.R., Milos, P.M., Agarwal, S., and Rao, A. (2011). Genomewide mapping of 5-hydroxymethylcytosine in embryonic stem cells. Nature 473, 394-397.

Pera, M.F., and Tam, P.P. (2010). Extrinsic regulation of pluripotent stem cells. Nature 465, 713-720.

Rodrigues, A.S., Pereira, S.L., Correia, M., Gomes, A., Perestrelo, T., and Ramalho-Santos, J. (2015). Differentiate or die: 3-bromopyruvate and pluripotency in mouse embryonic stem cells. PLoS One 10, e0135617.

Seisenberger, S., Andrews, S., Krueger, F., Arand, J., Walter, J., Santos, F., Popp, C., Thienpont, B., Dean, W., and Reik, W. (2012). The dynamics of genome-wide DNA methylation reprogramming in mouse primordial germ cells. Mol. Cell. 48, 849-862.

Shcherbina, A., Li, J., Narayanan, C., Greenleaf, W., Kundaje, A., and Chetty, S. (2019). Brief report: Cell cycle dynamics of human pluripotent stem cells primed for differentiation. Stem Cells 37, 1151-1157.

Singh, M.K., Singh, K.P., Kumar, D., Shah, R.A., Anand, T., Chauhan, M.S., Manik, R.S., Singla, S.K., and Palta, P. (2012). Buffalo (Bubalus bubalis) ES cell-like cells are capable of in vitro skeletal myogenic differentiation. Reprod. Domest. Anim. 48, 284-291.

Smith, A.G. (2001). Embryo-derived stem cells: Of mice and men. Annu. Rev. Cell. Dev. Biol. 17, 435-462.

Smith, A.G., Heath, J.K., Donaldson, D.D., Wong, G.G., Moreau, J., Stahl, M., and Rogers, D. (1988). Inhibition of pluripotential embryonic stem cell differentiation by purified polypeptides. Nature 336, 688-690.

Smith, Z.D., Chan, M.M., Mikkelsen, T.S., Gu, H., Gnirke, A., Regev, A., and Meissner, A. (2012). A unique regulatory phase of DNA methylation in the early mammalian embryo. Nature 484, 339-344.

Sroczynska, P., Lancrin, C., Pearson, S., Kouskoff, V., and Lacaud, G. (2009). In vitro differentiation of mouse embryonic stem cells as a model of early hematopoietic development. Methods Mol. Biol. 538, 317-334.

Su, T., and Waxman, D.J. (2004). Impact of dimethyl sulfoxide on expression of nuclear receptors and drug-inducible cytochromes P450 in primary rat hepatocytes. Arch. Biochem. Biophys. 424, 226-234.

Tahiliani, M., Koh, K.P., Shen, Y., Pastor, W.A., Bandukwala, H., Brudno, Y., Agarwal, S., Iyer, L.M., Liu, D.R., Aravind, L., and Rao, A. (2009). Conversion of 5-methylcytosine to 5hydroxymethylcytosine in mammalian DNA by MLL partner TET1. Science 324, 930-935.

Tay, Y., Zhang, J., Thomson, A.M., Lim, B., and Rigoutsos, I. (2008a). MicroRNAs to Nanog, Oct4 and Sox2 coding regions modulate embryonic stem cell differentiation. Nature 455, 1124-1128.

Tay, Y.M., Tam, W.L., Ang, Y.S., Gaughwin, P.M., Yang, H., Wang, W., Liu, R., George, J., Ng, H.H., Perera, R.J., Lufkin, T., Rigoutsos, I., Thomson, A.M., and Lim, B. (2008b). MicroRNA-134 modulates the differentiation of mouse embryonic stem cells, where it causes post-transcriptional attenuation of Nanog and LRH1. Stem Cells 26, 17-29.

Thaler, R., Spitzer, S., Karlic, H., Klaushofer, K., and Varga, F. (2012). DMSO is a strong inducer of DNA hydroxymethylation in pre-osteoblastic MC3T3-E1 cells. Epigenetics. 7, 635-651.

Vallier, L., and Pedersen, R.A. (2005). Human embryonic stem cells: An in vitro model to study mechanisms controlling pluripotency in early mammalian development. Stem Cell Rev. 1, 119-130.

Varum, S., Momcilovic, O., Castro, C., Ben-Yehudah, A., Ramalho-Santos, J., and Navara, C.S. (2009). Enhancement of human embryonic stem cell pluripotency through inhibition of the mitochondrial respiratory chain. Stem Cell Res. 3, 142-156.

Wang, J., Rao, S., Chu, J., Shen, X., Levasseur, D.N., Theunissen, T.W., and Orkin, S.H. (2006). A protein interaction network for pluripotency of embryonic stem cells. Nature 444, 364-368.

Williams, K., Christensen, J., Pedersen, M.T., Johansen, J.V., Cloos, P.A., Rappsilber, J., and Helin, K. (2011). TET1 and hydroxymethylcytosine in transcription and DNA methylation fidelity. Nature 473, 343-348.

Williams, R.L., Hilton, D.J., Pease, S., Willson, T.A., Stewart, C.L., Gearing, D.P., Wagner, E.F., Metcalf, D., Nicola, N.A., and Gough, N.M. (1988). Myeloid leukaemia inhibitory factor maintains the developmental potential of embryonic stem cells. Nature 336, 684-687.

Wu, H., D’Alessio, A.C., Ito, S., Xia, K., Wang, Z., Cui, K., Zhao, K., Sun, Y.E., and Zhang, Y. (2011). Dual functions of Tet1 in transcriptional regulation in mouse embryonic stem cells. Nature 473, 389-393.

Yamaguchi, S., Hong, K., Liu, R., Shen, L., Inoue, A., Diep, D., Zhang, K., and Zhang, Y. (2012). Tet1 controls meiosis by regulating meiotic gene expression. Nature 492, 443-447.

Ying, Q.L., and Smith, A.G. (2003). Defined conditions for neural commitment and differentiation. Methods Enzymol. $365,327-341$.

Yoshizawa, T., Watanabe, S., Hirose, M., Miyazaki, A., and Sato, N. (1997). Dimethylsulfoxide maintains intercellular communication by preserving the gap junctional protein 
connexin32 in primary cultured hepatocyte doublets from rats. J. Gastroenterol. Hepatol. 12, 325-330.

Youngblood, B.A., Alfano, R., Pettit, S.C., Zhang, D., Dallmann, H.G., Huang, N., and Macdonald, C.C. (2014). Application of recombinant human leukemia inhibitory factor (LIF) produced in rice (Oryza sativa L.) for maintenance of mouse embryonic stem cells. J. Biotechnol. 172, 67-72.

Zhu, G., Li, Y., Zhu, F., Wang, T., Jin, W., Mu, W., Lin, W., Tan, W., Li, W., Street, R.C., Peng, S., Zhang, J., Feng, Y., Warren, S.T., Sun, Q., Jin, P., and Chen, D. (2014). Coordination of engineered factors with TET1/2 promotes early-stage epigenetic modification during somatic cell reprogramming. Stem Cell Rep. 2, 253-261.

Address correspondence to: Myoung-Ok Kim Department of Animal Science and Biotechnology Kyungpook National University Sangju-si 37224 Gyeongsangbuk-do Korea

E-mail: ok4325@knu.ac.kr 